Teddy ARNAVIELLE

Université Paul Valéry-Montpellier III

E A 739 DIPRALANG

\title{
Centre et périphérie dans la CONJUgaison. Le CaS du GÉRONdif FRANÇAIS
}

\section{La conjugaison : problèmes de limites}

La conjugaison peut légitimement être présentée comme un ensemble prévisible : entendons que, une base verbale étant retenue, un ensemble complet de formes sera, au sens premier, décliné : leurs affinités formelles seront suffisantes pour qu'elles constituent la conjugaison du verbe sélectionné, organisée en sousensembles morphosyntaxiques appelés modes. C'est seulement dans le cas où l'une de ces formes acquiert des propriétés considérées comme non verbales qu'elle sera considérée comme dérivée : dériver n'est pas conjuguer. Ainsi on parlera d'infinitif dérivé en nom, ou d'infinitif substantivé : « le savoir, le dîner ».

Même les théories qui privilégient les disparités de la conjugaison ne remettent pas vraiment en question, en dépit de certaines apparences, ce modèle globalement unitaire ; ainsi

- de TESNIÈRE et de la translation (1959: 417-420) : si « le verbe transféré en substantif [...] est l'infinitif », il existe bien une « conjugaison du verbe voir ».

- de BALLY et sa transposition (1964 : 118) : l'infinitif, translaté ou transposé en substantif, reste attaché au paradigme verbal : «les participes, les infinitifs et le gérondif, qui transposent l'idée verbale respectivement en adjectif, en substantif et en adverbe [...] conservent la presque totalité de la syntaxe verbale [...], très voisins du verbe conjugué ».

- de BONNARD (1969 : 22-23) : l'infinitif est « virtuel en tant que verbe », en tant que l'expression du temps n'y est pas incluse.

- de MARTinet (1960 : 142) : les participes et les infinitifs sont dits dotés de «monèmes» qui ont «valeur d'affixe de dérivation». Mais, en 1979, les «modalités verbales »sont posées comme partagées par l'ensemble des modes de la conjugaison classique (même si Martinet et son équipe munissent de guillemets le terme de « conjugaison ») (91).

On n'est pas très loin, la théorisation en moins, de la chronogenèse de Guillaume, moyen élégant de traiter la gradualité des propriétés de l'ensemble verbal : «L'image du temps en puissance [...] correspond au mode quasi-nominal, l'infinitif et les participes » (MOIGNET, 1981:63).

Le paradigme n'est pas changé si l'on propose de renverser l'ordre de la chronogenèse : c'est la proposition que fait DE CARVALHO (Langages 2003: notamment 102., où la forme en -ant est définie comme un «nom post- ou déverbal adossé au verbe », sans que soit remis en question, ni ici ni ailleurs, le système de la conjugaison.

On n'entreprendra pas ici d'évaluer les mérites de ces différentes solutions, qui, toutes, s'installent, de façon plus ou moins affirmée, et non sans embarras, dans une opposition du dedans et du dehors, de l'intérieur et de l'extérieur. La 
commodité pédagogique n'est sans doute pas étrangère à ce choix, mais l'exigence de certaines au moins des théories proposées exclut que ce soit là la préoccupation dominante. Tout au plus notera-t-on que le sort réservé à cette forme en limite qu'est l'adjectif verbal (en - ant) n'est pas très clair : on préfère en général voir en lui un dérivé, dérivé franc pourrait-on dire parce que nettement hors du paradigme verbal donc, très lié à lui car la prévisibilité est forte, mais non totale : tout verbe ne peut donner naissance à un adjectif verbal - ni à un substantif en -ant plus ou moins lié audit adjectif - ou à un adjectif tiré de participe passé : "étant(s)étante(s) »n'existent pas, ni «voulant-voulante », même si leur création, sous la plume d'un écrivain, n'est pas exclue: autre chose est l'entrée dans l'usage. Position nettement théorisée par MoIGNET (1981 : 68) : «Dès que la forme en -ant quitte le système verbal, elle est dotée du genre et du nombre d'accord et devient un adjectif comme les autres. »

On ne peut, à cet égard, considérer comme satisfaisantes les analyses qui prétendent effacer, sur la base d'une affinité morphologique évidente, la distinction participe en -ant - adjectif verbal en -ant. WILMET (2007: 312) ne nous convainc pas qui considère comme négligeables les différences de compatibilité syntaxiques observables entre « un homme aimant beaucoup les enfants » et « un homme très aimant » : selon lui, c'est à tort que « l'enseignement bétonne la limite territoriale du 'participe présent' invariable - le participe-verbe et de l', adjectif verbal' variable - le participe-adjectif ». On ne peut, dans le même sens, considérer comme négligeable l'existence de formes en -ent («négligent », par exemple, face à «négligeant») qui sont purement adjectivales, donc jamais participes.

Le cas du participe passé et de l'adjectif de lui tiré est différent. Que le premier soit la forme la moins verbale de la conjugaison n'a pas à être souligné : la Grammaire du français classique et moderne de WAGNER - PINCHON (1962) avait tenté de marquer cette situation en proposant de réserver à ce participe la désignation « forme adjective du verbe » (235, notamment).

Ici, bien souvent, la morphologie de la variabilité n'est pas concluante : l'adjectif est toujours accordé, le participe qui lui correspond est soumis à une casuistique de la variation qui fait partie des grands classiques de la grammaire française. Le test de l'adverbe, en revanche, reste valide : «très » et «si », le duo d'adverbes réservé à la complémentation de l'adjectif, ne s'appliquent qu'au dérivé adjectival: «une veste très abîmée »; mais pas « une maison si transformée ». La frontière existe, nous l'avons rencontrée...

D'ailleurs, il ne semble pas que, dans ce cas comme dans celui de l'adjectif verbal, tout verbe puisse donner naissance à un adjectif ayant pour origine un participe passé : «voulu », ni «su », par exemple, n'existent. On laissera de côté les raisons sémantiques pouvant expliquer ces exclusions, que l'histoire en marche peut sans doute lever.

Par ailleurs, personne n'hésitera à poser hors de l'accueillante conjugaison les formes dérivées de base verbale en -able, -ible, -uble («lavable, lisible,...»), dont la prévisibilité est large, mais limitée : ni «*ayable », ni «*devable » ne sont disponibles, pour des raisons qu'il faudrait élucider. Si «-able » est un suffixe, «$a n t »$ ne l'est que pour former un adjectif verbal, «-é » pour produire un adjectif tiré (c'est-à-dire dérivé) de participe passé. 
Dans la terminologie fonctionaliste (ainsi chez MARTINET et al., 1979, 14, $3.19 \mathrm{~b}$ ), très clairement, si les participes sont des «syntagmes », les «adjectifs verbaux » sont des «synthèmes »: soit, respectivement, formes fléchies, formes dérivées.

\section{Unité et divisions}

Si l'on peut considérer que l'ensemble de la conjugaison existe, avec des limites établies conventionnellement, non sans incertitudes, comme on l'a souligné, malgré le caractère rassurant des traditionnels tableaux découpés en modes - terme qui, à la lettre, engage peu -, ce complexe est, de l'intérieur, profondément divisé. On rappellera brièvement, d'abord les éléments d'unification, puis les clivages.

Pour les premiers, l'opposition entre formes simples et formes composées est familière et très largement reconnue - parfois contestée, tout de même, comme on le notera un peu plus bas : la distinction des formes de non accompli et de celles d'accompli est capitale : «je mangeais - j'avais mangé ; manger - avoir mangé ».

Sur une aire plus réduite, la distinction des formes actives, simples, et des passives, composées, est notable: «mangeais - étais mangé ; manger - être mangé ».

D'un autre côté, le clivage est fort entre formes marquées de temporalité, et formes non marquées, ou entre modes temporels et non temporels, et il se double d'un autre, entre formes personnelles et formes non personnelles. Les appréciations, ici, peuvent varier, quant au degré de concordance entre ces deux propriétés :

- soit on considère que toute forme personnelle est aussi temporelle : c'est l'éventail, large, des formes d'indicatif, celui, plus réduit, de celles de subjonctif, celui, plus restreint encore, de celles d'impératif, que l'on gagne d'ailleurs à ramener soit à l'indicatif, pour l'essentiel, soit au subjonctif, pour une faible part (« sois, aie, veuille, puisse, sache») : la réalisation la plus achevée est ici la chronogenèse : au subjonctif, «l'image du temps » est «en devenir » (MOIGNET : 63) ;

- soit, position minoritaire, on pose que des formes personnelles sont temporelles, d'autres non (BONNARD, 1969 : 24) : le subjonctif est « un infinitif personnel » position inchangée par la suite.

MARTINET (1979: 100, 112) représente une position intermédiaire, qui accorde au subjonctif une faible capacité d'indication du temps; sur le tableau (100), : le monème « passé » est reconnu au subjonctif.

C'est le débat du subjonctif, sur lequel il n'y a en fait aucun moyen de trancher, ce mode étant toujours employé en dépendance (le sub-jonctif est mode de sub-ordination) : les positions prises seront fonction de l'importance accordée au co-texte ; à ce titre, elles correspondent à un choix théorique fort.

Ces oppositions morpho-sémantiques se doublent d'oppositions proprement syntaxiques : la personne permet à la forme verbale de fonctionner avec un sujet, lequel est déjà inclus dans la forme verbale, sous la marque affixale, et, de surcroît, extériorisé par un indice pronominal (obligatoire en français comme clitique) ou développé par un identificateur nominal ou pronominal non clitique. 
Modèle couramment admis, avec quelques variations portant notamment sur le poids du clitique, non reconnu comme porteur de fonction par TOURATIER, notamment (2005: 177 et sv.), traité comme un «syntagme » en Grammaire générative, au moins dans des versions anciennes. L'absence de la marque de personne, caractéristique de certains «modes », empêche l'accès à la prédication avec sujet (on sera libre de parler de prédication sans sujet personnel dans « Et tous de se récrier »), sinon, évidemment dans les assemblages d'auxiliation ( «Il vient de sortir ») et prédispose les formes à des fonctions de complémentation - y compris dans la fonction de sujet, qui peut être vue comme un complément obligatoire : « Sortir me plairait ; j'aimerais sortir ».

Indépendante de ces oppositions, une autre, moins connue, et souvent présentée indirectement: celle entre le bloc constitué des formes personnelles (indicatif, impératif, subjonctif) et des participes présent et passé, d'une part; d'autre part, l'infinitif, auquel on peut adjoindre le gérondif (forme.en -ant prépositionnelle, en français). Mode de signification et fonctionnement nominaux d'une part, mode de signification et fonctionnements adjectivaux et verbaux d'autre part. La psychomécanique de Guillaume parle respectivement d' «incidence externe » et d' «incidence interne » (MOIGNET, 1981: 14 et sv.), entendant que l'infinitif, comme le nom, n'ont pas de support hors d'eux-mêmes, alors que les autres modes trouvent leur support hors d'eux-mêmes. Ce que nous traduirons en disant que seul l'infinitif, dans cet ensemble, a vocation d'actant, avec les fonctions substantives liées à ce statut. Proche de lui à cet égard, le gérondif pourra être actant, mais seulement au sens large, comme circonstant, plus périphérique (temps, moyen, etc.). De l' «incidence interne » à l' «autonomie » telle que définie par MARTINET (1985: 124), il semble qu'il n'y ait pas loin ; notons-le au passage, car il ne semble pas que ce rapprochement inattendu ait jamais été fait : «Un adjectif comme rapide est aussi autonome que l'adverbe vite dans ce sens qu'il implique non seulement une qualité, mais le fait que cette qualité s'applique à une entité déterminée. [...] C'est l'absence d'autonomie qui semblerait caractériser une classe particulière, les noms. »

Dans le détail, la situation est en fait plus complexe. En effet, si l'infinitif est bien du régime nominal (support interne) et le participe du régime adjectival (support externe), les modes personnels tiennent, en fait, des deux. Qu'est-ce, en effet, qu'une forme d'indicatif sinon un terme qui, à la fois « dit quelque chose sur quelque chose » et inclut cette « chose » même dont il est « dit quelque chose »? Si la base verbale « dit quelque chose », la désinence personnelle « est ce quelque chose »; le thème est à la fois dans le verbe et hors de lui, alors que le rhème n'est que dans le verbe. En psychomécanique, on pourra ainsi qualifier le verbe personnel de «sujet-prédicat» (MOIGNET, 1981 : 91).

On notera au passage, en écho à une remarque présentée plus haut, qu'il est possible de refuser l'analyse qui oppose formes composées et formes simples : la tentative a été faite par ZEMB (1972 : 101-118) qui entreprend de montrer que le deuxième terme de la composition, infinitif, participe, peut être traité comme un complément du premier terme : l'analyse morphologique s'efface devant l'analyse syntaxique. L'avantage de la proposition est de frapper de caducité la question irritante des verbes auxiliaires et semi-auxiliaires, auxquels on peut ajouter les coverbes - à moins que ce dernier terme, assez mal assuré, ne couvre l'ensemble 
(voir WILMET, 2007 : 338 et sv.). Si on adopte ce point de vue, c'est un facteur d'unification qui s'affaiblit sans disparaître : la valeur d'accompli est évidemment conservée, c'est seulement la façon de l'inscrire dans les formes qui est modifiée. Du même coup, la conjugaison est notablement simplifiée, tous les assemblages « composés » étant renvoyés à la syntaxe : « les classiques tables de conjugaison sont très éclectiques, souvent superfétatoires » (ZEMB 1972 :118).

\section{Vers le centre et la périphérie}

Ces rappels et ces précisions étant faits, il reste à les situer par rapport à la distinction du centre et de la périphérie.

Le centre peut être ce lieu de la conjugaison où sont réunies le plus grand nombre de propriétés considérées comme verbales; le verbe accompli, fini en somme - les deux fois aux deux sens du mot - sorte de noyau dur, ou d'optimum. A la périphérie, le moins verbal, doté de propriétés minimales.

La perspective est au fond celle d'une morphologie, mieux : d'une morphosémantique cumulative.

On peut aussi, avançant d'un pas, considérer que ce minimum n'en est même pas un et «exclure » ces «peu-disants» de la conjugaison; ainsi, le cercle sera réduit, comme la concentricité. A cette tentation, il n'est pas habituel, on l'a vu, de succomber, parfois de peu, et non sans embarras. Si on ne fait pas ce choix, donc, on pourra distinguer un centre du centre, correspondant à l'indicatif, largement et lui seul complètement temporalisé.

Le subjonctif et l'impératif, chacun à sa façon, seront à distance de ce centre : de deux façons différentes, l'une marquée par une forte défectivité en temps et en personnes pour le second, mais dont les formes sont souvent indistinctes de celles de l'indicatif ; l'autre, le premier, avec des spécificités morphologiques assez bien marquées, qu'on ne rappellera pas ici.

En raffinant, on sera tenté de «placer » à petite distance du centre les emplois non strictement temporels, dits parfois inactuels, de l'indicatif : formes à valeur hypothétique, à ancrage temporel large, à la façon du subjonctif : "S'il était là aujourd'hui/demain, nous sortirions ». C'est au fond, avec d'autres raisons, ce que fait la tradition scolaire, non éteinte, traitant souvent le conditionnel comme un mode, au moins quand il n'est pas un «futur du passé », mais oubliant l'imparfait irréel, ce que ne fait pas Bonnard qui, tentant de concilier tradition pédagogique et exactitude sémantique, distingue des «Modes de l'irréel», au prix, choisi, d'une réduction de la pertinence des traits formels (1981 : 233-4).

Il est également loisible de poser comme centre les mêmes formes personnelles cette fois parce que leur syntaxe est liée à la prédication, le mécanisme de base, le plus simple et aussi le plus fondamental, fondateur de phrases; c'est une extension à la syntaxe pragmatique de la centralité morphosémantique privilégiée ci-dessus.

A la périphérie figurent alors les formes qui, dénuées de la capacité à faire prédication, permettent, en revanche, une complexification de la syntaxe par la complémentation, la détermination ; c'est ce que note MARTIN (1991 : 256) : «s'il est vrai que dans la construction sémantique de la langue, l'infinitif - tout comme le subjonctif - constitue un avant par rapport à l'indicatif » (contre DE CARVALHO, 
2003 : supra) «,... il est vrai aussi qu'au plan syntaxique les phrases les plus simples sont des phrases à l'indicatif et que l'infinitif et le subjonctif n'y entrent qu'à mesure que, par les enchâssements, sa complexité va s'acroissant. »

Pour nous limiter aux formes non-personnelles, leur morphologie simple facilite leur extension syntaxique : le cas le plus net est évidemment celui de l'infinitif, plurifonctionnel dans des conditions comparables à celles du nom, sans abandonner la rection verbale.

C'est cette voie que suit, avec sa démarche propre, la grammaire générative, au moins dans ses premiers développements, supposant que l'infinitif implique par transformation un effacement du sujet : la forme personnelle est en somme posée en préalable.

D'un autre point de vue, pourra être considéré comme centre le lot commun à l'ensemble des_formes verbales : leur régime, par lequel, à deux extrémités de la conjugaison, indicatif et participe commandent les mêmes compléments : le participe passé lui-même n'échappe pas à cette parenté, même s'il la manifeste surtout avec le secours d'un auxiliaire: "J'ai fini ce livre»; mais l'elliptique «Fini ce livre» est possible. A ce lot commun, des formes ajouteraient des propriétés secondes : intégration de la personne, de la temporalité, permettant d'autres aptitudes syntaxiques : c'est la périphérie, vue comme arrangement qui complexifie.

C'est - comment s'en étonner ? - le point de vue qui prime, et qui, au fond, rend le choix impossible :

- sur le champ morphologique, la richesse de marques de l'indicatif à la fois permet son rôle syntaxique majeur de verbe avec sujet, et lui interdit d'autres emplois : c'est par la structure de la proposition subordonnée que ce mode participe au système des actants, par la procédure de la nominalisation ou de l'adjectivisation: «Il vient, je le sais » : juxtaposition de propositions : deux prédications ; «Je sais qu'il vient» (ou «Je le sais, qu'il vient»): mise en subordination qui utilise comme élément nominalisé l'assemblage sujet-verbe, et en fait un actant complément d'objet. Le verbe est repoussé à la périphérie, comme "prédicatoïde », dans la terminologie de Martinet.

- sur le champ syntaxique, la pauvreté de marques de l'infinitif et du participe - même si leurs désinences peuvent être analysées comme des «orienteurs » de fonction - les ouvre à des emplois variés, le premier surtout, la prédication personnelle étant exclue ; mais la complexification de la phrase leur est largement due, ce qui n'est que rarement souligné, tant la structure de la propostion pèse avec force sur les esprits. Au plan didactique, le constat peut présenter quelque intérêt : on proscrira toute présentation qui ferait des modes non personnels du verbe inachevé, en manque si l'on veut; pas d'inachèvement, mais une ouverture ; pas de scission entre l'infinitif-verbe et l'infinitif-nom, découpage désastreux en ce qu'il décompose ce qui et une synthèse riche.

On peut ne pas se satisfaire de cette solution, qui ne résout pas l'embarras (mais faut-il absolument le résoudre ?). C'est la position adoptée par Hjelmslev, ce grand linguiste à notre sens pas assez consulté aujourd'hui, notamment pour ses travaux de syntaxe. C'est à une véritable révolution que nous convie le fondateur de la glossématique, d'abord en nous invitant à reconsidérer les fondements de nos analyses morphologiques et syntaxiques. 
Reportons-nous aux Essais linguistiques (Editions de Minuit, 1971), pour en détacher quelques formules particulièrement fortes dans le très important chapitre «Le verbe et la phrase nominale » (195-198 notamment) :

«Les morphèmes dits «verbaux» appartiennent à la phrase prise dans son ensemble, et non au verbe seul »?

«Le verbe est [...] une base nue. [...] Dès le moment où un verbe [...] revêt une caractéristique qui lui est propre, et qui n'appartient pas à la phrase, le verbe se révèle comme un nom : c'est le gérondif (amandum), c'est le participe, c'est l'infinitif, et [...] c'est le nom verbal [...] En un mot, c'est le verbe infini, au sens large. »

« La limite entre verbe et nom tend à s'effacer [...] tout verbe fini est un nom potentiel. [...] il ne nous reste qu'une simple base qui admet deux variantes : une variante nominale et une variante verbale ».

«La transformation du pseudo verbe en nom donne comme résultat le nom verbal ou le verbe infini. »

La netteté des affirmations rend leur commentaire superflu.

Notons seulement ceci : avec Hjelmslev, l'opposition centre-périphérie, qui continue à nous occuper, prend une tout autre signification : le centre, comme espèce de générateur, c'est la base (base $=$ centre). Mais cette base n'existe que pour être complétée (ce qui n'est pas le cas dans les autres hypothèses précédemment évoquées), c'est un «potentiel» (en un sens différent de celui où Hjelmslev parle du «verbe fini » comme «nom potentiel», sinon un «virtuel») d'identité neutre ; il est achevé, réalisé par les éléments de périphérie, dans deux directions :

- nominale : «verbe infini » (infinitif, participe, gérondif)

- phrastique - adjectif proposé par nous ; les morphèmes portent sur la phrase, puisqu'ils permettent, notamment, la prédication : «verbe fini »- qui est dit «nom potentiel» parce qu'on peut toujours, avec lui, (re)faire du nom, dans le sens (supra) proposé par Hjelmslev, celui de «nom d'action», au sens large incluant participe (et gérondif).

Permettons-nous une critique : la notion de «nom », si largement alléguée, n'est en fait pas bien définie, au point de paraître presque signifier « mot», tout simplement; en fait, tout ce qui ne «prédique » pas est dit nom - d'autant que la distinction «nom »- « verbe » est fortement relativisée (on parle indistinctement de «nom verbal » ou de «verbe infini »), en même temps que le «verbe » est réduit à la base. Ce qui, d'ailleurs, autre objection, ne manifeste pas une grande cohérence. Plus largement, la notion de partie de discours est, dans cette démarche, fortement relativisée.

Alors, que faire de Hjelmslev, finalement ? On peut retirer de cette lecture dérangeante et stimulante une conscience plus aiguë de l'importance de la prédication (personnelle, au moins), comme phénomène lié à des morphèmes, mais qui porte en fait sur toute la phrase. Ce serait seulement le caractère un peu radical de la position du linguiste danois qui serait objet de critique. 


\section{L’ensemble « non fini »}

Après l'ensemble de la conjugaison, on s'attachera au sous-ensemble du verbe non fini, autrement dit les formes assez mal qualifiées nominales du verbe.

On le choisit comme micro-système dans le système de la conjugaison, et on s'interroge dans ce cadre sur le couple centre et périphérie. Cette démarche est indépendante des différents choix présentés plus haut, en gros : centre $=$ formes personnelles : ou centre $=$ formes non personnelles, à partir de deux points de vue différents, de deux conceptions du rapport centre-périphérie.

Dans ce sous-ensemble, où le centre ? où la périphérie ? Si on met du côté du périphérique que l'on peut dire extérieur adjectif verbal et adjectif issu de participe passé (et évidemment les dérivés en -ble), que dire de l' « intérieur »?

On y trouve des formes (des modes, si l'on veut) à vocations différentes :

- substantivale (cas de l'infinitif): sa diversité de fonctions, d'essence nominale, peut permettre de voir en lui un fort périphérique, par expansion, si l'on veut : ce serait le plus périphérique dans le (déjà) périphérique : on a déjà évoqué l'incidence interne, et, en couplage, l' « autonomie » de Martinet.

- adjectivale (les participes) : c'est un degré de moins dans la périphéricité, du point de vue des potentialités syntaxiques, où le participe joint le verbe personnel sur l'incidence externe.

De la gradualité, toutefois, de la forme en -ant, du côté du plus, au participe passé, pour le moins : formulé autrement: celui-ci est plus périphérique par ses variations formelles, l'absence d'auxiliation du même au même (* «été parti »; mais : «j’ai été parti »).

Avec le cas fort embarrassant du gérondif, qui tient d'un domaine, substantival, et de l'autre, adjectival (par le morphème -ant partagé). Sa périphéricité est inscrite dans sa structure même, avec préposition : c'est un phénomène largement acquis, et stabilisé par l'histoire, pas très bien expliqué, ni en synchronie, ni en diachronie (voir cependant ARNAVIELLE, à paraître, KLEIBER) ; à quoi s'ajoute une résistance à l'auxiliation d'accompli (d'une espèce différente de celle du participe passé - cf. supra). On va y revenir.

Pour compliquer: on a un périphérique du point de vue de la prédicativité (ou : prédication) : le participe en -ant (ou comment ne pas faire phrase ?), ajouté au blocage attributif: ni «*Criant trop fort, ce garçon» (prédication sans verbe personnel; la forme en -ant serait rhème), ni « Il est criant trop fort, ce garçon » (prédication avec forme en -ant attribut) ne sont possibles ; la situation est très différente pour le participe passé, moins pour le gérondif, avec cependant la contrainte de construction qu'entraîne la préposition, qui limite la prédication sans la bloquer (on est plutôt en fait devant un circonstant de prédication : «En lisant, en écrivant »).

Donc, des croisements de propriété, qui compliquent beaucoup l'analyse ; on ne conclura pas, ramené à la conclusion que prime, à nouveau, la question de point de vue, ou de l'angle de vue.

Tout au moins, consacrons davantage notre attention à ce mal connu qu'est le gérondif, ce qui ne manquera pas de nous ramener à la question de la géographie, ou de la topographie, de l'ensemble. 


\section{Et le gérondif ?}

A différents égards, le gérondif français, dont on rappellera qu'il a peu à voir avec les gérondifs anglais ou espagnol (DE CARVALHO, 2003 : 105 et sv.) au point qu'on doit remettre en question une terminologie source de confusion, pose des problèmes d'intégration dans l'ensemble non fini. D'où, quant à la question de son placement, des positions fort différentes, rappelées brièvement ici.

Martinet y voit une «variante de l'infinitif»(1979: 114), solution radicalement syntaxique - et morpho-sémantique, d'une certaine façon (valeurs circonstancielles partagées, appuyées sur les prépositions).

La solution en apparence la plus simple, parce que fondée sur une reconnaissance formelle, consiste à voir dans le gérondif une construction, prépositionnelle, du participe en -ant : ainsi WILMET (2007, «Sic transit $[\ldots] »)$ n'hésite pas à traiter de la même façon les oppositions «aimant »- « en aimant », d'une part, et « aimer »- «d'aimer » (infinitif sans et avec préposition), de l'autre.

C'est au fond, sans le dire, ce que font la grande majorité des grammaires $-\mathrm{y}$ compris, curieusement et non sans un certain illogisme, Bonnard et Martinet, qui ne vont pas jusqu'au bout dans leurs tableaux ou leurs schémas (Martinet, symptomatiquement, ne propose pas de tableau de conjugaison).

Analyse en fait superficielle, argumentée chez Wilmet seul, lequel ne voit pas que, si la préposition ne fait que confirmer le caractère nominal de l'infinitif, « en », seule préposition retenue - ce qui ne doit pas manquer d'intriguer - ne peut pas naturellement sanctionner le caractère substantival d'un... participe, plutôt considéré, habituellement et à bon droit, comme du côté de l'adjectival...

Peut-être peut-on se tirer d'affaire en notant que la coexistence de «en » et d'un adjectif s'observe ailleurs, sans que cet adjectif soit forcément substantivé : « en (très) grand, gros, bref» etc.; et il existe, il est vrai, une locution «en définitive », dans laquelle « définitive » ne peut être autre chose qu'adjectif.

La psychomécanique adopte la position souple selon laquelle il existe une « forme en -ant », adjectivale, mais qui, en certains emplois, peut «basculer » du côté du substantival, sans que le détail du mécanisme soit exposé.

Poussant à bout ce choix, nous avons émis ailleurs (ARNAVIELLE, à paraître) l'hypothèse que la forme en -ant ne serait, au fond, en soi ni substantivale ni adjectivale, et que seuls les emplois en décideraient, en l'orientant ici (adjectif) ou là (substantif).

Ce qui remettrait en question la nature prépositionnelle de «en », vu plutôt comme un complémenteur, au sens et avec les applications retenues par RIEGEL PELlAT - Rioul (1994 : 474, 496 ; cf. ARNAVIELle, à paraître).

Ainsi, la forme en -ant serait une bonne candidate au statut de centre, en tant que terme de soi faiblement conditionnée, alors que l'infinitif, résolument substantival, et le participe II ( «passé»), nettement adjectival, seraient en périphérie.

Rappelons ici que, dans le schéma de la chronogenèse, la forme en -ant est un «après » de l'infinitif, et un « avant » du participe II (MOIGNET, 1980 : 65-70, notamment), en vertu de sa composition aspectuelle: incidence + décadence, cumulées, alors que la seule incidence caractérise l'infinitif, la décadence seule le participe; cette répartition harmonieuse serait sans doute à discuter (cf. 
ARNAVIELLE 2003 : 52-53). Si nous l'acceptons provisoirement, le gérondif, quant à lui, à penchant substantival, sera, dans cette perspective, attiré vers l'infinitif, et figurera un périphérique du participe en -ant, dont il partage le morphème final. $\mathrm{A}$ quoi s'ajoute la périphéricité que lui confère sa forme composée, au sens le plus large et non technique du terme (cf. KLEIBER, 2007) pour une discussion làdessus) : nous préférons dire : d'allure composée.

Certes, le rapprochement évoqué avec la chronogenèse trouve vite ses limites, car l'approche est très différente, mais la rencontre est intéressante qui, dans les deux cas, pose la forme en -ant comme la moins spécialisée, ce qui peut être vu comme un trait de centralité.

Si l'on se livre à une revue rapide du sémantisme, on est notamment attiré par la valeur dite de « reformulation» (KLEIBER, 2007); en voici quelques exemples :

« Reformulation »:

Très populaire au Japon, ce jeu a mis un pied en Europe en débarquant en Angleterre (Le Monde, 16-3-6)

Et Sablier n'attendit pas le retour du général au pouvoir pour en tirer la conclusion en reprenant sa liberté (id.)

Le ministère de l'Intérieur commet une erreur grossière en associant automatiquement salafisme et terrorisme (Libération, 14-3-6)

L'entraîneur portugais sanctionnait John Cole et Shaun Wright-Philips en les remplaçant (L'Equipe, 20-3-6)

Cette valeur est intéressante pour notre propos parce que : la «préposition » (mieux : complémenteur) fonctionne comme un élément qui : à la fois pose à côté, établit une distance, et, d'autre part, permet le passage d'une continuité sémantique, un peu à la façon du «de » qui figure dans «L'idée de partir» («l'idée du-de départ»), où le deuxième terme développe le premier; la différence est peut-être que, avec l'infinitif ou le nom, le terme est forcément générique (d'où une démarche partie-tout), ce qui ne paraît pas être le cas, ou alors pas aussi nettement, avec le gérondif. Ce point serait cependant à examiner de plus près, en tenant compte que, pour l'infinitif et le nom, le support est un nom, pour le gérondif un verbe. Ce qui change pas mal de choses, car le verbe n'a pas la capacité d'abstraction du nom (de type abstrait s'entend).

En tout cas, on est devant un point de rencontre entre infinitif et gérondif : en quoi on peut voir - ne perdons pas notre fil - un « recentrage » de ce dernier.

Faut-il établir un lien entre le développement historique, vraisemblable mais qui reste à mesurer, de la valeur de « reformulation », où l'on peut voir une forme de grammaticalisation, d'une part, et, d'autre part, les progrès, mal évalués par les descripteurs, de la forme composée non passive du gérondif ?

Un regard d'abord sur les grammaires et leurs rituels tableaux de conjugaison. Le plus souvent, il n'y a pas de case réservée pour le gérondif, abrité derrière le 
participe en -ant alors même que ses spécificités de structure et de valeurs sont reconnues : Wilmet le «cache » sous le participe et s'en explique.

Ainsi font aussi BONNARD (1981 - un peu contradictoire), WAGNER Pinchon (1962), CheVAlier - Arrivé - Peytard - Benveniste (1964), RIEGEL - PELLAT - RIOUL, etc.

Plus précise - et l'on reconnaît là l'esprit scrupuleux de Michel Arrivé -, la Grammaire d'aujourd'hui (1986 : 143) le dit « à peu près inusité » et ne le retient dans les tableaux de conjugaison qu'avec un point d'interrogation et une note («forme rare »), mais à part du participe. Position confortée par la remarque un peu étonnante (488): «Le gérondif passé n'est guère qu'une curiosité de grammairien : introduit par la préposition en, il ne saurait marquer l'antériorité. Les quelques exemples qu'on en trouve font apparaître une valeur d'accompli ». Tant il est vrai que les grammairiens, même parmi les meilleurs, voient parfois la langue non comme elle est, mais comme elle devrait être...

GREVISSE (1980 : 771, 778, etc.) tout en prévoyant un casier pour le gérondif, laisse vide le «tiroir » composé. Mais dans GREVISSE - GoOSSE (2008 : 1143), le tiroir est cette fois garni d'une forme dite « rare », illustrée par trois exemples, du $\mathrm{XIX}^{\mathrm{e}}$ siècle (Balzac, Goncourt) et du $\mathrm{XX}^{\mathrm{e}}$ (Proust), qui apparaissent comme des liberté d'écrivain.

Inusité, presque incongru, le gérondif composé ? Ne serait-ce pas une idée reçue, circulant de grammaire en grammaire sans être vraiment réexaminée ? On n'a pas grand mal, en fait, à réunir un corpus, récent, tout entier, il est vrai, constitué de textes de presse, dont certains restituent des paroles. Qu'on en juge par cette courte liste, simple échantillon dans une collecte de plusieurs dizaines de formes :

Le Mans est donc sacré, vingt-quatre ans après, en ayant pris des chemins sinueux (L'Equipe, 19-6-6)

En ayant fait un mandat sur la $2^{\mathrm{e}}$, et si demain il est élu sur la $1^{\mathrm{e}}$, il maîtrisera et surtout activera tous ses réseaux (Midi libre, 12-6-7)

Un chef de parti pouvait fustiger les chômeurs en ayant toute sa vie été luimême fonctionnaire (Le Monde, 18-2-5)

En ayant gardé cette intégrité, je peux me sentir incorruptible (TV Magazine, 14/20-5-6)

Et le centre ? Et la périphérie ? Pour la première fois, sans doute allons-nous pouvoir trancher : l'aire est étroite, mais l'enseignement n'est pas dénué d'intérêt.

La structure du gérondif, de quelque façon qu'on l'explique, pose un problème, et fait de lui, si on ne se contente pas de solutions simplificatrices (souvent benoîtement appuyées sur des motifs pédagogiques), un objet atypique, en français. A cette marginalité participe, ou plutôt participait, car la langue change, sa non accession ou son accessiblité problématique à la composition avec auxiliaire. Et voilà que le français vivant est en train de réduire cette différence : 
réduire aux deux sens de diminuer - c'est l'état de langue actuel -, et d'effacer état de langue de demain, peut-être.

On allèguera, comme principe d'explication, l'analogie, très légitimement : que le participe ait un couple simple-composé entraîne que son proche, le gérondif, se dote d'un couple analogue. La solution satisfera aussi bien ceux qui relient le gérondif au participe, démarche qui vient d'être suivie, que ceux qui l'associent à l'infinitif : des deux côtés, c'est la même pression analogique qui, décidément, fait entrer le gérondif dans le rang de la conjugaison.

Il resterait à évaluer l'importance sémantique de cette évolution (pas d'analogie sans pression du sens) : nous n'en avons pas ici le loisir. Permettonsnous seulement de mettre en relation, en cumul même, ce progrès morphosémantique et l'importance prise par la valeur de reformulation, analysée peu avant, qui correspond pour nous à un stade avancé d'allègement sémantique de « en », et à son altération identitaire de préposition réorientée en complémenteur. C'est toujours de la même opération de «mise au pas » du gérondif qu'il s'agit: rabotage de la préposition, affectation répandue d'un auxiliaire. Ou, allons plus loin, peut-être trop loin, effacement partiel ou total de cette espèce de compositon, en un sens large, que constitue l'assemblage de «en » et de la forme en -ant, au profit de l'entrée dans le régime courant de la composition par auxiliation. Ceci ne valant pas cela, il ne semble pas qu'il y ait de rapport sémantique entre les deux évolutions. Retenons seulement l'opération de normalisation à quoi correspondent ces deux modifications; opération qui, en dernier ressort, a bien à voir avec la sémantique ; une conjugaison harmonisée, comme lissée, recentrée, pour reprendre notre fil, ce sont bien, en fait, des potentialités sémantiques d'ensemble remodelées...

\section{Conclusion}

Nous poserons, pour terminer, et en élargissant la perspective, que la conjugaison, en diachronie longue, paraît marquée à la fois par une démarche d'élargissement, d'expansion: le cas des formes surcomposées, même si leur succès historique est très inégal, est connu ; et par une démarche de resserrement, d'unification, dans certaines limites bien entendu: on rappellera ici la perte acquise de la variabilité du participe en -ant (voir pour détail ARNAVIELLE, 1997 : 13 et passim notamment); les incertitudes de variabilité du participe passé («la route que j'ai pris»). Ces deux mouvements peuvent être vus comme un effacement, acquis ou en cours (mais la norme résiste) de caractères non verbaux, favorisant une meilleure insertion de ces formes verbales peu affirmées. Qui, par ailleurs, ne se souvient que le gérondif (prépositionnel) a longtemps balancé entre le nom et le verbe (là aussi, voir Arnavielle, 1997 : 84 et sv.), hésitation favorisée par la bivalence de la préposition «en », laquelle a d'ailleurs longtemps alterné avec d'autres prépositions (encore ARNAVIELLE, 1997 : 75 et sv.) ? 


\section{BIBLIOGRAPHIE}

ARNAVIELlE Teddy (1997), Le morphème -ant: unité et diversité. Etude historique et théorique, Louvain-Paris, Peeters.

ARNAVIELlE Teddy (2003), Le participe, les formes en -ant: positions et propositions.

Langages 149, Participe présent et gérondif, Paris, Larousse, p. 37-54.

ARNAVIELlE Teddy, La forme en -ant: unité d'abord. (A paraître in Revue des langues romanes, 2010).

ARrIVÉ Michel, GADET Françoise, GALMICHE Michel (1986), La grammaire d' d'aujourd'hui. Guide alphabétique de linguistique française, Paris, Flammarion.

BALly Charles (1964, $4^{\mathrm{e}}$ éd., $1^{\mathrm{e}}$ éd. 1932), Linguistique générale et linguistique française, Berne, Francke.

BONNARD Henri (1969), Guillaume, il y a vingt ans, Langue française 1, La syntaxe, Paris, Larousse, p. 21-35.

BONNARD, Henri (1981), Code du français courant, Paris, Magnard, 1981.

Chevallier Jean-Claude, ArRivé Michel, PeYtard Jean, BlancheBENVENISTE Claire, Grammaire du français contemporain, Paris, Larousse, 1964.

De Carvalho Paulo (2003), «Gérondif», «participe présent» et «adjectif déverbal» en -ant en morphosyntaxe comparative, Langages 149, Paris, Larousse, p. 100-124.

GREVISSE Maurice (1980,11 éd.), Le Bon usage, Paris-Gembloux, Duculot.

GREVISSE Maurice et Goosse André (2008, $14^{\mathrm{e}}$ éd.), Le Bon usage, Bruxelles, De Boeck.

HJELMSLEV Louis (1971), Essais linguistiques, Paris, Editions de Minuit.

KLEIBER Georges (2007), En passant par le gérondif avec mes (gros) sabots, Cahiers Chronos, 19. L'accès à cet article nous a été fourni par son auteur, ici remercié, mais sans la pagination.

MARTIN Robert (1992, $2^{\mathrm{e}}$ éd.), La syntaxe, in POTTIER Bernard (éd.), Les sciences du langage en France au $X X^{e}$ siècle, Paris, Peeters-SELAF, p. 235266.

MARTinet, André (1967), Eléments de linguistique générale, Paris, A. Colin, collection «U».

MARTINET André et al. (1979), Grammaire fonctionelle du français, Paris, CredifDidier.

MARTINeT André (1985), Syntaxe générale, Paris, A. Colin, collection «U ».

MOIGNET Gérard (1980), Systématique de la langue française,Paris, Klincksieck.

RIEGEL Martin, PELlat Jean-Christophe, Rioul René (1994), Grammaire méthodique du français, Paris, P. U. F.

TESNIÈRE Lucien (1959), Eléments de syntaxe structurale, Paris, Klincksieck.

TOURATIER Christian (2005), Analyse et théories syntaxiques, Aix-en-Provence, Publications de l'Université de Provence.

WAGNER Robert-Léon et PINCHON Jacqueline (1962), Grammaire du français classique et moderne, Paris, Hachette. 
Wilmet Marc (2007, 4 éd., $1^{\mathrm{e}}$ éd. 1997), Grammaire critique du français, Bruxelles, De Boeck.

WILMET Marc (2007), Sic transit gloria mundi : à propos de quelques survivances latines en grammaire française, in Représentations du sens linguistique, II, P. Bouchard, I. Evrard, E. Vocaj. (édd.), Bruxelles, De Boeck, «Champs linguistiques », p. 235-236.

ZEMB Jean-Marie (1972), Métagrammaire. La proposition, Paris, O. C. D. L.

\section{SUMMARY}

We shall defend here the idea that the French (prepositional) Gerund is in the contemporary language on the way of evolution which reduces its "peripheral" character, observable in particular in its difficult compatibility with auxiliary verbs "avoir" and "être, to "refocus" it in the conjugation, where the compound forms acquire a more important place. We can suppose that this movement is in connection with the reduction of the character of preposition of "en", for the benefit of a status of simple "complémenteur" (complementizer), what shows the expansion of the value of "reformulation". 